\title{
ON COLLINEAR CLOSED ONE-FORMS
}

\author{
IRINA GELBUKH
}

(Received 4 January 2011)

\begin{abstract}
We study one-forms with zero wedge-product, which we call collinear, and their foliations. We characterise the set of forms that define a given foliation, with special attention to closed forms and forms with small singular sets. We apply the notion of collinearity to give a criterion for the existence of a compact leaf and to study homological properties of compact leaves.
\end{abstract}

2010 Mathematics subject classification: primary 57R30; secondary 58A10.

Keywords and phrases: differential one-form, singular set, foliation, compact leaves, cup-product.

\section{Introduction and statement of the main results}

We consider a closed oriented $n$-dimensional manifold $M$ and a smooth one-form $\alpha$ on it with the singular set $\operatorname{Sing} \alpha$. This form defines on $\operatorname{Supp} \alpha=M \backslash \operatorname{Sing} \alpha$ a foliation $\mathcal{F}_{\alpha}$, which can be extended to the whole of $M$ as a singular foliation $\overline{\mathcal{F}}_{\alpha}$ (Definition 5.2). We shall study the conditions for other forms $\beta$ to define the same foliation: $\mathcal{F}_{\beta}=\mathcal{F}_{\alpha}$ or $\overline{\mathcal{F}}_{\beta}=\overline{\mathcal{F}}_{\alpha}$.

We call forms $\alpha, \beta$ collinear, denoted by $\alpha \| \beta$, if $\alpha \wedge \beta=0$ [10]. Collinear one-forms appear in many problems of theoretical physics, for example in general relativity: type I vacuum solutions with aligned Papapetrou fields [6] or triplet ansatz $[2,11]$. Ranks (of group of the periods) of collinear Morse forms (closed oneforms with nondegenerate singularities) have been studied in [10].

On the intersection of their supports, collinear forms are proportional (Lemma 3.3). Moreover, on this set they share important properties, integrability in particular (Proposition 3.6), and, if they are integrable, the foliation. Thus the set of the forms that define the same foliation $\mathcal{F}_{\alpha}$ are those that (obviously) have the corresponding support and are collinear with $\alpha$ (Theorem 3.7).

The relation of collinearity is reflexive and symmetric, but generally not transitive (Figure 1). For Morse forms, however, collinearity is an equivalence relation [10]; we generalise this fact to one-forms with nowhere dense singular sets (Proposition 3.8). In this case, integrability of the form is a class invariant and the singular foliation uniquely identifies integrable classes (that is, it is a class invariant which is different

(C) 2011 Australian Mathematical Publishing Association Inc. 0004-9727/2011 \$16.00 
for different classes). Similarly, for one-forms with a common support, collinearity is an equivalence relation (Corollary 3.5), integrability is a class invariant, and the foliation uniquely identifies integrable classes.

Closed forms, that is, $d \alpha=0$, are an important class of integrable one-forms. They are especially interesting because they allow the use of cohomology techniques. In particular, $\alpha \wedge \beta=0$ implies $[\alpha] \smile[\beta]=0$, where $\smile: H^{1}(M, \mathbb{Z}) \times H^{1}(M, \mathbb{Z}) \rightarrow$ $H^{2}(M, \mathbb{Z})$ is the cup-product and $[\alpha]$ is the cohomology class; this is used in our study of foliations defined by closed forms (Theorem 7.3). In addition, closed one-forms define an important class of foliations - foliations without holonomy; moreover, any codimension-one foliation without holonomy is topologically equivalent to a foliation defined by a closed one-form [19]. For closed one-forms, the singular foliation $\overline{\mathcal{F}}_{\alpha}$ is a good approximation of the foliation $\mathcal{F}_{\alpha}$ (Lemmas 5.3-5.6).

For closed collinear one-forms, the intersection of their supports consists of entire leaves of both forms (Corollary 4.3). In particular, no leaf of one form can intersect the boundary of the support of the other form (Proposition 4.2).

Our main result states that for closed one-forms with small enough singular sets, their foliations coincide if and only if the forms are collinear (Theorem 6.5); this was already known for Morse forms [10]. Note that, unlike the case in Theorem 3.7, coincidence of supports is no longer required but is instead guaranteed by the conditions.

Namely, for closed one-forms with at most $(n-2)$-dimensional singular sets, $\mathcal{F}_{\alpha}=\mathcal{F}_{\beta}$ holds if and only if $\alpha \| \beta$ (Theorem 6.5); note that for such forms $\alpha \| \beta$ implies $\operatorname{Supp} \alpha=\operatorname{Supp} \beta$. We mean here the topological (covering) dimension (Definition 2.1), since Sing $\alpha$ is generically not a submanifold.

The condition on Sing $\alpha$ can be relaxed: for closed one-forms with at most $(n-1)$-dimensional, that is, nowhere dense (Lemma 2.3), singular sets, $\overline{\mathcal{F}}_{\alpha}=\overline{\mathcal{F}}_{\beta}$ holds if and only if $\alpha \| \beta$ (Theorem 6.5). Further generalisations are as follows: for (not necessarily closed) one-forms with $\operatorname{dim} \operatorname{Sing} \alpha \leq 0, \alpha \| \beta$ implies $\overline{\mathcal{F}}_{\alpha}=\overline{\mathcal{F}}_{\beta}$. (Proposition 6.2); for closed one-forms (irrespective of their singular sets) $\overline{\mathcal{F}}_{\alpha}=\overline{\mathcal{F}}_{\beta}$ implies $\alpha \| \beta$ (Proposition 6.4).

The notion of collinearity is useful for the study of foliations defined by closed one-forms, as can be illustrated by the following examples.

Farber et al. [4, 5] gave a necessary condition for the existence of a compact leaf in the foliation defined by a so-called transitive Morse form, in terms of the cup-product. We generalise this condition in terms of collinearity to an arbitrary closed one-form and improve it to a criterion (Theorem 7.2).

In [10], it was shown that the topology of a foliation defined by a Morse form of the maximum possible rank for the given $M, \operatorname{rk} \alpha=b_{1}(M)$ (the first Betti number), is connected with the structure of the cup-product, namely, $c(\alpha) \leq \operatorname{rk}$ ker $\smile$, where $c(\alpha)$ is the maximum number of homologically independent compact leaves of $\mathcal{F}_{\alpha}$, an important value in the theory of foliations defined by Morse forms [8, 9]. We use the notion of collinearity to generalise this fact to arbitrary closed one-forms (Theorem 7.3). 
The paper is organised as follows. In Section 2, we give various conditions for Sing $\alpha$ to be small, in terms of covering dimension. In Section 3, we introduce collinear forms and consider some of their properties. In Section 4, we study the supports of closed collinear one-forms. In Section 5, we define the notion of singular foliation and show that it is well behaved for closed forms. In Section 6, we prove our main result on the characterisation of the set of closed forms with small supports that define the same foliation, as an equivalence class of collinear forms. Finally, in Section 7 , we use the notion of collinearity to study homological properties of compact leaves of a foliation defined by a closed one-form.

\section{Singular set}

We consider smooth one-forms on a closed oriented $n$-dimensional manifold $M$. The one-form $\alpha$ is a smooth section of the cotangent bundle of $M$, that is, it assigns to each point $x \in M$ a linear functional $\alpha_{x}: T_{x} M \rightarrow \mathbb{R}$. Denote

$$
\text { Sing } \alpha=\left\{x \in M \mid \alpha_{x}=0\right\} .
$$

Obviously, Sing $\alpha \subseteq M$ is closed and the support $\operatorname{Supp} \alpha=M \backslash \operatorname{Sing} \alpha$ is open. Generically, Sing $\alpha$ is not a submanifold.

To characterise a subset of $M$ that is not necessarily a submanifold, we use the topological (covering) dimension.

Definition 2.1 [12]. The covering dimension $\operatorname{dim} X$ of a topological space $X$ is the minimum value $n$ such that every open cover of $X$ has an open refinement in which no point is included in more than $n+1$ elements.

For instance, $\operatorname{dim} \emptyset=-1$; the covering dimension of a simplicial complex is the maximum dimension of its simplices; and $\operatorname{dim}\left(S^{2} \vee S^{1}\right)=2$. If $Y \subseteq X$, then $\operatorname{dim} Y \leq \operatorname{dim} X$. The covering dimension of a manifold coincides with its conventional dimension [12, Corollary 1, Theorem IV.3]. In particular, the following remark holds.

REMARK 2.2. If $X \subseteq M$ and $X \supseteq i \mathbb{R}^{k}$ ( $i$ is an inclusion), then $\operatorname{dim} X \geq k$.

For $X \subseteq M, \quad \operatorname{dim} X \leq n-1$ if and only if the interior $\operatorname{int}(X)=\emptyset \quad[12$, Theorem IV.3]; in particular, the covering dimension of a leaf of a foliation is $n-1$. If $\operatorname{dim} X \leq n-2$, then $X$ does not locally divide $M$ [12, Theorem IV.4].

Thus $\operatorname{dim} \operatorname{Sing} \alpha \leq n=\operatorname{dim} M$; obviously, $\partial \operatorname{Supp} \alpha \subseteq \operatorname{Sing} \alpha=M \backslash \operatorname{Supp} \alpha$.

LEMMA 2.3. For a one-form $\alpha$, the following conditions are equivalent:

(i) Sing $\alpha$ is nowhere dense in $M$;

(ii) $\operatorname{Supp} \alpha$ is dense in $M$;

(iii) $\partial \operatorname{Supp} \alpha=\operatorname{Sing} \alpha$;

(iv) $\operatorname{int}(\operatorname{Sing} \alpha)=\emptyset$;

(v) $\operatorname{dim} \operatorname{Sing} \alpha \leq n-1$. 


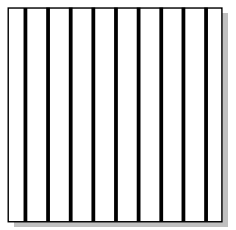

$\alpha=d x$

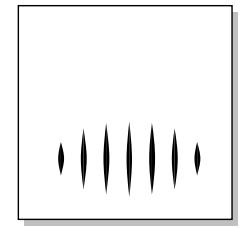

$\omega_{1}=f(x, y) d x$

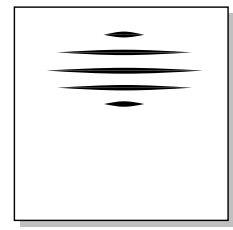

$\omega_{2}=g(x, y) d y$

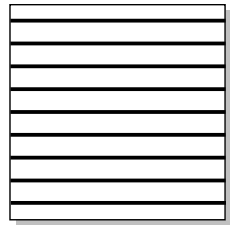

$\beta=d y$

FIgURE 1. Chain of collinear forms on a 2-torus. Note that $S\left(\omega_{1}, \omega_{2}\right)=\emptyset ; f$ and $g$ are bump functions.

PROOF. (ii) $\Leftrightarrow$ (iii). $Y \subseteq X$ is open and dense if and only if $\partial Y=Y^{C}$, the complement. (iii) $\Leftrightarrow$ (iv). $\operatorname{int}(Y)=\emptyset$ if and only if $Y=\partial\left(Y^{C}\right)$. (iv) $\Leftrightarrow(\mathrm{v}) \cdot \operatorname{dim} Y<\operatorname{dim} X$ if and only if $\operatorname{int}(Y)=\emptyset[12$, Theorem IV.3]. (iv) $\Leftrightarrow$ (i). Sing $\alpha$ is closed.

\section{Collinear one-forms}

Definition 3.1. Two differential forms $\alpha, \beta$ are called collinear if $\alpha \wedge \beta=0$; we denote this as $\alpha \| \beta$.

The term is motivated by Lemma 3.3 below.

REMARK 3.2. The set of $x \in M$ at which $\alpha \| \beta$ is closed.

Denote $S(\alpha, \beta)=\operatorname{Supp} \alpha \cap \operatorname{Supp} \beta$; it is open. The collinearity relation is reflexive and symmetric but not necessarily transitive: obviously, $S(\alpha, \beta)=\emptyset$ implies $\alpha \| \beta$, so any two forms $\alpha, \beta$ are connected via two forms $\omega_{j}$ such that $\alpha\left\|\omega_{1}, \omega_{1}\right\| \omega_{2}$, and $\omega_{2} \| \beta$, even if $\alpha \wedge \beta \neq 0$ (see Figure 1; see [18]). Therefore collinearity is generally not an equivalence relation. In what follows, however, we shall consider various conditions under which collinearity is an equivalence.

LEMMA 3.3. Let $\alpha, \beta$ be one-forms. The following conditions are equivalent:

(i) $\alpha \| \beta$;

(ii) $\alpha=f(x) \beta$ on $\operatorname{Supp} \beta$;

(iii) $\beta=g(x) \alpha$ on $\operatorname{Supp} \alpha$.

On $S(\alpha, \beta)$, the functions $f(x)$ and $g(x)$ are smooth and nonvanishing, with $f(x) g(x)=1$.

PROOF. (ii) or (iii) $\Rightarrow$ (i) is obvious.

(i) $\Rightarrow$ (ii) and similarly (iii). Consider on $\operatorname{Supp} \beta$ a smooth vector field $\xi$ with nonvanishing $\beta\left(\xi_{x}\right)$. Choose

$$
f(x)=\frac{\alpha\left(\xi_{x}\right)}{\beta\left(\xi_{x}\right)},
$$

which, by collinearity, does not depend on the choice of $\xi$. Indeed, for any other such field $\xi^{\prime}$ collinearity gives

$$
(\alpha \wedge \beta)\left(\xi_{x}, \xi_{x}^{\prime}\right)=\alpha\left(\xi_{x}\right) \beta\left(\xi_{x}^{\prime}\right)-\alpha\left(\xi_{x}^{\prime}\right) \beta\left(\xi_{x}\right)=0 .
$$

Thus $\alpha=f(x) \beta$ on $\operatorname{Supp} \beta$; in addition, $f(x) \neq 0$ on $S(\alpha, \beta)$. 
So on $S(\alpha, \beta)$ collinear forms are proportional, thus the term. However, this does not necessarily hold on the whole $M$ : for instance, any two forms with nonintersecting supports are collinear, but not proportional.

Corollary 3.4. On Supp $\beta, \alpha \| \beta$ and $\beta \| \gamma$ imply $\alpha \| \gamma$.

Corollary 3.5. Consider a set $A$ of one-forms on $M$ such that $S(\alpha, \beta)=S \subseteq M$ is the same for any $\alpha, \beta \in A$; for instance, $\operatorname{Supp} \alpha=S$ for any $\alpha \in A$. Then the collinearity is an equivalence relation on $A$.

On $S(\alpha, \beta)$, collinear forms share important properties. Consider an integrable form $\alpha$; this defines a foliation $\mathcal{F}_{\alpha}$ on $\operatorname{Supp} \alpha$.

Proposition 3.6. Let $\alpha$ be an integrable one-form. Then for a one-form $\beta$ it holds that $\beta \| \alpha$ if and only if on $S=S(\alpha, \beta)$ it is also integrable and $\left.\mathcal{F}_{\beta}\right|_{S}=\left.\mathcal{F}_{\alpha}\right|_{S}$.

By $\left.\mathcal{F}\right|_{\mathcal{S}}$ we understand a foliation of $S$ whose leaves are path-connected components of $\gamma \cap S, \gamma \in \mathcal{F}$.

PROOF. Let $\alpha \| \beta$. By the Frobenius theorem, $\alpha$ is integrable if and only if $\alpha \wedge d \alpha=0$. By Lemma 3.3, on $S$ it holds that $\beta=f(x) \alpha$, so $\beta \wedge d \beta=0$ and thus $\beta$ is also integrable. The foliations coincide since $\{\alpha=0\} \equiv\{f \alpha=0\}$.

Assume now $\mathcal{F}_{\alpha}=\mathcal{F}_{\beta}$ on $S(\alpha, \beta)$. Outside $S(\alpha, \beta)$, the forms are obviously collinear. Consider $x \in S(\alpha, \beta)$ and $\xi_{x}, \eta_{x} \in T_{x} M=\left\langle n_{x}, T_{x} \gamma\right\rangle$, where $\gamma \in \mathcal{F}_{\alpha}$ is the leaf that contains $x$ and $n_{x} \in T_{x} M \backslash T_{x} \gamma ; \xi_{x}=a n_{x}+\xi_{x}^{\prime}, \eta_{x}=b n_{x}+\eta_{x}^{\prime}$ for some $a, b \in \mathbb{R}$ and $\xi_{x}^{\prime}, \eta_{x}^{\prime} \in T_{x} \gamma$. Since $\left.\alpha\right|_{T_{x} \gamma}=\left.\beta\right|_{T_{x} \gamma}=0$,

$$
(\alpha \wedge \beta)\left(\xi_{x}, \eta_{x}\right)=\alpha\left(a n_{x}\right) \beta\left(b n_{x}\right)-\alpha\left(b n_{x}\right) \beta\left(a n_{x}\right)=0,
$$

that is, $\alpha \| \beta$.

Therefore, collinear forms are either both integrable or both nonintegrable on $S(\alpha, \beta)$, that is, integrability on $S$ is a class invariant under the equivalence relation from Corollary 3.5. Integrable one-forms $\alpha, \beta$ are collinear if and only if $\mathcal{F}_{\alpha}=\mathcal{F}_{\beta}$ on $S(\alpha, \beta)$, that is, $\left.\mathcal{F}\right|_{S}$ uniquely identifies an integrable class under this equivalence relation.

We have obtained a characterisation of the set of forms defining a given foliation $\mathcal{F}$ as the equivalence class, under the collinearity relation, of forms with the corresponding support. In particular, we obtain the following theorem.

THEOREM 3.7. Given a foliation $\mathcal{F}=\mathcal{F}_{\alpha}$ of a one-form $\alpha$, the forms defining $\mathcal{F}$ are one-forms collinear with $\alpha$ and having the same support.

The latter condition automatically holds and thus can be omitted from Theorem 3.7 in the class of closed forms with small enough singular sets; this is formulated below as part of Theorem 6.5 .

Forms with small singular sets give another important class of forms on which collinearity is an equivalence relation. 

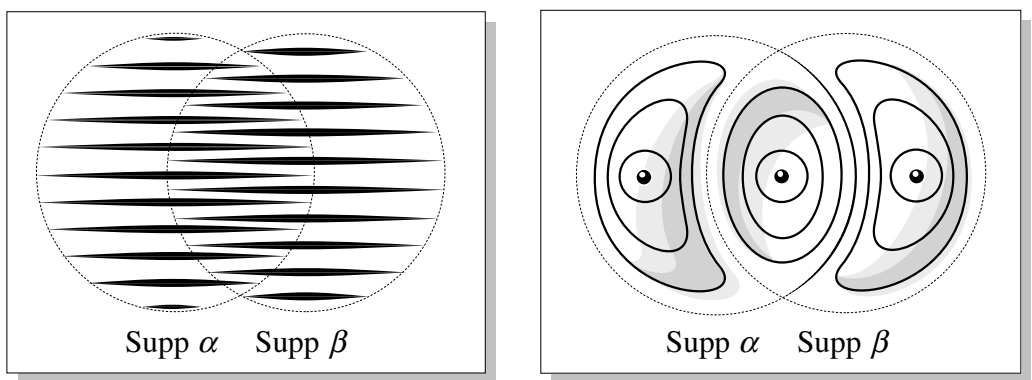

FIGURE 2. Left: partial intersection of leaves is possible only for nonclosed collinear forms; right: intersecting leaves of closed collinear forms coincide.

PROPOSITION 3.8. On the set of one-forms with nowhere dense singular sets (see Lemma 2.3), collinearity is an equivalence relation.

PROOF. It is sufficient to prove transitivity. Suppose $\alpha \| \beta$ and $\beta \| \gamma$, but $\alpha \not \lambda \gamma$ at $x \in M$ and thus, by Remark 3.2, on some open $U \neq \emptyset$. By Corollary 3.4, $U \subseteq \operatorname{Sing} \beta$, which is a contradiction.

In this case, integrability on the whole of $M$ is a class invariant (by Proposition 3.6 and the fact that $\alpha \wedge d \alpha$ is continuous) but the foliation is not, as can be seen in Figure 3. For such closed forms, however, the singular foliation introduced below in Definition 5.2 is a class invariant uniquely identifying an equivalence class. This is also formulated below as part of Theorem 6.5.

\section{Supports of closed collinear one-forms}

In what follows, we consider an important class of integrable forms: closed oneforms. Properties of the supports of closed collinear one-forms prove to be connected with their foliations.

LEMMA 4.1. Let $\alpha, \beta$ be closed one-forms and $\alpha=f(x) \beta$ on $\operatorname{Supp} \beta$. Then $f(x)$ is constant on leaves of $\mathcal{F}_{\beta}$.

This follows by direct calculation from the equation $d \alpha=d f \wedge \beta=0$.

By Proposition 3.6, leaves of collinear integrable one-forms $\alpha, \beta$ coincide on $S=S(\alpha, \beta)$, that is, $\left.\mathcal{F}_{\alpha}\right|_{S}=\left.\mathcal{F}_{\beta}\right|_{S}$. The following proposition shows that if both forms are closed, then leaves of one form cannot intersect the boundary of the support of the other form; see Figure 2.

Proposition 4.2. Let $\alpha, \beta$ be closed collinear one-forms and $\gamma_{\alpha} \in \mathcal{F}_{\alpha}$. Then $\gamma_{\alpha} \cap$ Supp $\beta \neq \emptyset$ implies $\gamma_{\alpha} \subset \operatorname{Supp} \beta$ and, moreover, $\gamma_{\alpha} \in \mathcal{F}_{\beta}$ (and similarly for $\operatorname{Sing} \beta$ ).

PROOF. By Lemma 3.3, on Supp $\alpha$ it holds that $\beta=f(x) \alpha$. Let $\gamma_{\alpha} \cap \operatorname{Supp} \beta \neq \emptyset$. If $\gamma_{\alpha} \not \subset \operatorname{Supp} \beta$, then there exists $x_{0} \in \gamma_{\alpha} \cap \operatorname{Sing} \beta$, so $f\left(x_{0}\right)=0$. By Lemma 4.1, we 


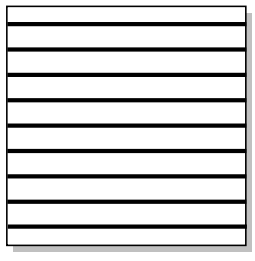

$\alpha=d y$

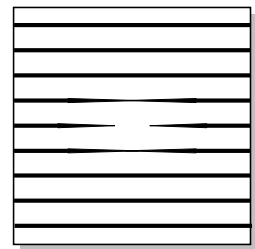

$\beta=\left(x^{2}+y^{2}\right) d y$

FIGURE 3. One-forms on a 2-torus (far from the singularity, the coefficient is assumed to be 1).

have $f(x)=0$ on $\gamma_{\alpha}$, that is, for all $x \in \gamma_{\alpha}$ it holds that $\beta_{x}=0$, thus $\gamma_{\alpha} \subset \operatorname{Sing} \beta$; this is a contradiction. By Proposition 3.6, $\gamma_{\alpha} \in \mathcal{F}_{\beta}$.

The condition for the forms to be closed is important. Indeed, on a torus $M=T^{2}$, consider $\alpha=d y$ and $\beta=\left(x^{2}+y^{2}\right) d y$ locally (while far from the singularity, assume this coefficient to be 1); see Figure 3. Then $\operatorname{Supp} \alpha=M$ and $\operatorname{Supp} \beta=M \backslash\{0\}$; $\gamma=\{y=0\} \in \mathcal{F}_{\alpha}$ intersects both $\operatorname{Supp} \beta$ and $\operatorname{Sing} \beta=\{0\}$.

Corollary 4.3. Let $\alpha, \beta$ be closed collinear one-forms; $\gamma_{\alpha} \in \mathcal{F}_{\alpha}$ and $\gamma_{\beta} \in \mathcal{F}_{\beta}$. Then $\gamma_{\alpha} \cap \gamma_{\beta} \neq \emptyset$ implies $\gamma_{\alpha}=\gamma_{\beta}$. In particular,

$$
S(\alpha, \beta)=\bigcup_{\gamma \in \mathcal{F}_{\alpha} \cap \mathcal{F}_{\beta}} \gamma .
$$

Indeed, by Proposition 4.2, $\gamma_{\alpha}, \gamma_{\beta} \subset S(\alpha, \beta)$; then, by Proposition 3.6, $\gamma_{\alpha}=\gamma_{\beta}$.

In other words, leaves of $\alpha$ and $\beta$ coincide if they intersect, and the intersection of their supports consists of entire leaves of both forms.

\section{Singular foliation of a closed form}

Let $\alpha$ be an integrable one-form. Its foliation is defined only on $\operatorname{Supp} \alpha$, namely, the following remark holds.

REMARK 5.1. A foliation $\mathcal{F}_{\alpha}$ is a decomposition of Supp $\alpha$ into leaves: two points $p, q \in \operatorname{Supp} \alpha$ belong to the same leaf $\gamma$ if and only if there exists a smooth path $s:[0,1] \rightarrow \operatorname{Supp} \alpha, s(0)=p, s(1)=q$ such that $\alpha(\dot{s}(t)) \equiv 0, t \neq 0,1$.

This notion can be extended to the whole of $M$ to define a so-called singular foliation. In [4], a singular foliation for Morse forms (their singular sets are finite) is defined as in Remark 5.1 by substituting $M$ for Supp $\alpha$. For arbitrary integrable forms we, however, prefer to relax the smoothness condition.

Definition 5.2. A singular foliation $\overline{\mathcal{F}}_{\alpha}$ is a decomposition of $M$ into leaves: two points $p, q \in M$ belong to the same leaf $\tilde{\gamma}$ if and only if there exists a continuous path $s:[0,1] \rightarrow M, s(0)=p, s(1)=q$ such that on $s^{-1}(\operatorname{Supp} \alpha) \backslash\{0,1\}$ it is differentiable and $\alpha(\dot{s}(t)) \equiv 0$. 


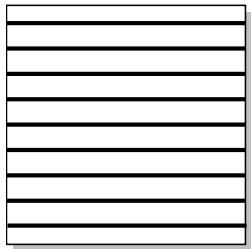

$\alpha=d y$

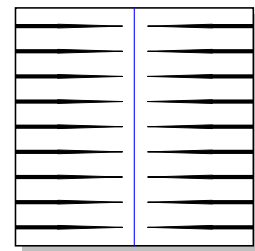

$\beta=x^{2} d y$

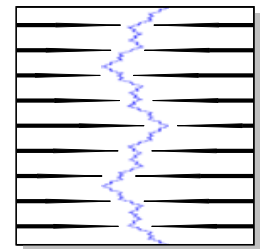

$\theta=f_{W}(x, y) d y$

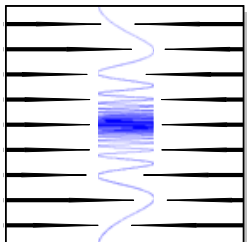

$\zeta=f_{S}(x, y) d y$

FIGURE 4. Smooth integrable one-forms on a 2-torus $M=T^{2}$ (the coefficients are assumed to be 1 far from the singular set). Sing $\theta$ is the graph of the Weierstrass function, which is continuous but nowhere differentiable. As a closed subset of $M$, it is the set of zeros of a smooth function $f_{W}$ [13, Theorem 1.5]. Note that this path-connected set does not contain any nontrivial differentiable path $s:[0,1] \rightarrow M$. The function $f_{S}$ is obtained similarly from the closed topologist's sine curve; it is connected but not pathconnected.

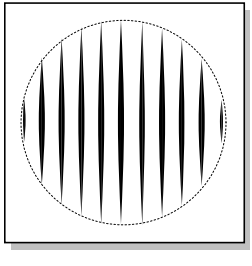

$\alpha=f(x, y) d x$

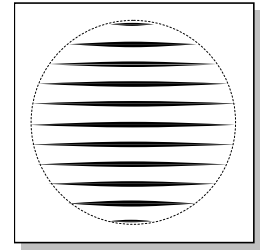

$\beta=f(x, y) d y$

FIgURE 5. Nonclosed one-forms on a 2-torus $M=T^{2} ; f$ is a bump function. Supp $\alpha=\operatorname{Supp} \beta$ and $\overline{\mathcal{F}}_{\alpha}=\overline{\mathcal{F}}_{\beta}=\{\tilde{\gamma}\}, \tilde{\gamma}=M$, but $\alpha \not \beta$.

In Figure $4, \overline{\mathcal{F}}_{\alpha}=\mathcal{F}_{\alpha} ; \overline{\mathcal{F}}_{\beta}=\{\tilde{\gamma}\}, \tilde{\gamma}=M$; and $\mathcal{F}_{\zeta}=\left\{\tilde{\gamma}_{1}, \tilde{\gamma}_{2}\right\}, \tilde{\gamma}_{1}=S^{1}$. With the relaxed smoothness condition, as in Definition 5.2, we have $\overline{\mathcal{F}}_{\theta}=\overline{\mathcal{F}}_{\beta}$, while if we required the path to be smooth on $M$, we would have $\overline{\mathcal{F}}_{\theta}=\overline{\mathcal{F}}_{\alpha}$, which is, perhaps, a matter of personal taste.

For any $\gamma \in \mathcal{F}_{\alpha}$, if $\gamma \cap \tilde{\gamma} \neq \emptyset$, then $\gamma \subseteq \tilde{\gamma}$, that is, a singular leaf of $\overline{\mathcal{F}}_{\alpha}$ consists of entire leaves of $\mathcal{F}_{\alpha}$ and path-connected components of Sing $\alpha$.

While leaves $\gamma$ of a usual foliation $\mathcal{F}$, which have the same dimension $\operatorname{dim} \gamma=n-1$, leaves $\tilde{\gamma}$ of a singular foliation $\overline{\mathcal{F}}$ can have different dimensions $0 \leq \operatorname{dim} \tilde{\gamma} \leq n$. For instance, a centre singularity is a zero-dimensional singular leaf; if $\operatorname{int}(\operatorname{Sing} \alpha) \neq \emptyset$, then there exists $\tilde{\gamma} \in \overline{\mathcal{F}}_{\alpha}$ with $\operatorname{dim} \tilde{\gamma}=n$ (see Lemma 2.3). By Remark 5.1, if $\operatorname{dim} \tilde{\gamma} \neq$ $n-1$, then $\tilde{\gamma} \cap \operatorname{Sing} \alpha \neq \emptyset$; in particular, $\operatorname{dim} \tilde{\gamma} \leq n-2$ implies that $\tilde{\gamma} \subseteq \operatorname{Sing} \alpha$ is a path-connected component of Sing $\alpha$.

The notion of a singular foliation as defined by Definition 5.2, while well defined, is rather counter-intuitive for nonclosed forms. As seen in Figure 4, for a leaf $\tilde{\gamma} \in \overline{\mathcal{F}}_{\alpha}$, it is possible that $\operatorname{dim}(\tilde{\gamma} \cap \operatorname{Supp} \alpha)=n$ or even $\tilde{\gamma}=M$ with $\operatorname{Supp} \alpha \neq \emptyset$. Thus for a curve $s(t) \subseteq \tilde{\gamma}$, it is possible that $\alpha(\dot{s}(t)) \neq 0$. Two noncollinear forms with Supp $\alpha=\operatorname{Supp} \beta$ can have $\overline{\mathcal{F}}_{\alpha}=\overline{\mathcal{F}}_{\beta}$, as in Figure 5 . 
For closed forms, however, the notion of $\overline{\mathcal{F}}_{\alpha}$ is quite intuitive and is a good approximation of $\mathcal{F}_{\alpha}$, as we show in the rest of this section. The form $\beta$ in Figure 4 shows that closedness is important in Lemmas 5.3-5.6.

LEMMA 5.3. Let $\alpha$ be a closed one-form, $\tilde{\gamma} \in \overline{\mathcal{F}}_{\alpha}$, and $s(t) \subseteq \tilde{\gamma}$ a differentiable curve. Then $\alpha(\dot{s}(t)) \equiv 0$.

Proof. Consider $F(x)=\int_{0}^{x} \alpha(\dot{s}(t)) d t$. Since $s \subseteq \tilde{\gamma}$, for any $x \in[0,1]$ there exists a path $u_{x}:[0,1] \rightarrow M$ connecting $s(x)$ and $s(0)$ such that $\int_{0}^{1} \alpha\left(\dot{u}_{x}(t)\right) d t=0$. Thus $F(x) \in P$, where $P=\left\langle\int_{z} \alpha \mid z \in H_{1}(M)\right\rangle_{\mathbb{Z}}$ is the group of periods. Since $P$ is finitely generated and thus at most countable while $F$ is continuous, we have $F \equiv$ const and thus $\alpha(\dot{s}(t)) \equiv 0$.

Corollary 5.4. Let $\alpha$ be a closed one-form and $\tilde{\gamma} \in \overline{\mathcal{F}}_{\alpha}$. Then $\operatorname{int}(\tilde{\gamma}) \subseteq \operatorname{Sing} \alpha$.

COROllary 5.5. For a closed one-form, Sing $\alpha$ is nowhere dense if and only if $\operatorname{dim} \tilde{\gamma} \leq n-1$ for any $\tilde{\gamma} \in \overline{\mathcal{F}}_{\alpha}$.

Lemma 5.6. For a closed form, $\left.\overline{\mathcal{F}}_{\alpha}\right|_{\operatorname{Supp} \alpha}=\mathcal{F}_{\alpha}$.

By $\left.\overline{\mathcal{F}}\right|_{S}$ we understand, again, a decomposition of $S$ into path-connected components of $\tilde{\gamma} \cap S, \tilde{\gamma} \in \overline{\mathcal{F}}$.

Proof. Consider a path-connected component $X \subseteq \tilde{\gamma} \cap \operatorname{Supp} \alpha$ and a point $x \in X$. Then $x \in \gamma$ for some $\gamma \in \mathcal{F}_{\alpha}$, so $\gamma \subseteq X$. For any $x^{\prime} \in X \backslash x$ there exists a curve $s(t) \subseteq X \subseteq \tilde{\gamma}, s(0)=x$ and $s(1)=x^{\prime}$. By Lemma 5.3, $\alpha(\dot{s}(t))=0$; by Remark 5.1, $x^{\prime} \in \gamma$. Thus $X=\gamma$.

\section{Collinear forms with small singular sets}

We shall show that for closed one-forms with small enough singular sets, collinearity implies coincidence of their singular foliations or, in the case of even smaller singular sets, coincidence of their (conventional) foliations and, in particular, their supports.

Recall that $\operatorname{dim} X$ stands for the covering dimension. On the set of one-forms with $\operatorname{dim} \operatorname{Sing} \alpha \leq n-1$, collinearity is an equivalence relation (Proposition 3.8).

LEMMA 6.1. Let $\alpha \| \beta$ be integrable one-forms, with $\operatorname{dim} \operatorname{Sing} \alpha \leq 0$. Then for any $\tilde{\gamma}_{\alpha} \in \overline{\mathcal{F}}_{\alpha}$ there exists $\widetilde{\gamma}_{\beta} \in \overline{\mathcal{F}}_{\beta}$ such that $\tilde{\gamma}_{a} \subseteq \tilde{\gamma}_{\beta}$.

PROOF. Let $p, q \in \tilde{\gamma}_{\alpha}$, that is, there exists $s:[0,1] \rightarrow M, s(0)=p, s(1)=q$ such that $\alpha(\dot{s}(t))=0$. Let us show that $\beta(\dot{s}(t))=0$, then $\widetilde{\gamma}_{\alpha} \subseteq \widetilde{\gamma}_{\beta}$.

Suppose $\beta(\dot{s}(t)) \neq 0$ for some $t \in[0,1]$. Since $\operatorname{dim} \alpha \leq 0$, by Remark 2.2, for any small neighbourhood $U(t)$ there exists $t^{\prime} \in U$ such that $s\left(t^{\prime}\right) \in \operatorname{Supp} \alpha$. By Lemma 3.3, in a small neighbourhood of $s\left(t^{\prime}\right)$ we have $\beta=f(x) \alpha$, thus $\beta\left(\dot{s}\left(t^{\prime}\right)\right)=0$. We obtain $\beta(\dot{s}(t))=0$; this is a contradiction. 


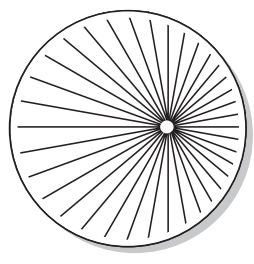

$\alpha$

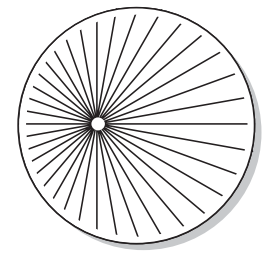

$\beta$

FIgURE 6. Nonclosed one-forms $\alpha \not X \beta$ on $M=S^{2}$, each one with two singularities; $\operatorname{dim} \operatorname{Sing} \alpha=$ $\operatorname{dim} \operatorname{Sing} \beta=0$, Supp $\alpha \neq \operatorname{Supp} \beta$, but $\overline{\mathcal{F}}_{\alpha}=\overline{\mathcal{F}}_{\beta}=\{\tilde{\gamma}\}, \tilde{\gamma}=M$.

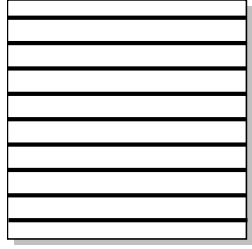

$\alpha=d y$

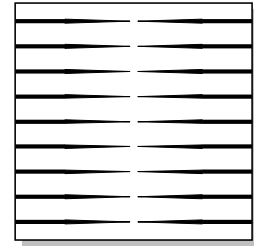

$\beta=x^{2} d y$

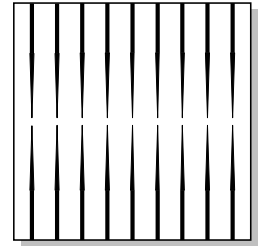

$\theta=y^{2} d x$

FIGURE 7. One-forms on a 2-torus $M=T^{2}$ (far from the singular set, the coefficients are equal to 1 ). Sing $\beta=S^{1}$, Sing $\theta=S^{1}, \overline{\mathcal{F}}_{\beta}=\overline{\mathcal{F}}_{\theta}=\{M\}$.

Proposition 6.2. Let $\alpha \| \beta$ be integrable one-forms with $\operatorname{dim} \operatorname{Sing} \alpha \leq 0$ and $\operatorname{dim} \operatorname{Sing} \beta \leq 0$. Then $\overline{\mathcal{F}}_{\alpha}=\overline{\mathcal{F}}_{\beta}$.

The converse is not true: Figure 6 shows two 'meridional' forms $\alpha$ Xl $\beta$ on $S^{2}$ with different 'poles'; however, $\overline{\mathcal{F}}_{\alpha}=\overline{\mathcal{F}}_{\beta}$.

The condition $\operatorname{dim} \operatorname{Sing} \alpha \leq 0$ in Lemma 6.1 and Proposition 6.2 cannot be relaxed to a higher value; see the forms $\alpha$ and $\beta$ in Figure 7. Note that an example analogous to $\beta$ can be constructed in any dimension $n$, with $\operatorname{dim} \operatorname{Sing} \beta=1$.

Lemma 6.3. Let $\alpha \| \beta$ be closed one-forms and $\operatorname{dim} \operatorname{Sing} \alpha \leq n-2$. Then $\operatorname{Sing} \alpha \subseteq$ $\operatorname{Sing} \beta$.

PRoOF. Suppose there exists a point $x \in \operatorname{Sing} \alpha \cup \operatorname{Supp} \beta$. Consider $\gamma_{\beta} \in \mathcal{F}_{\beta}$ such that $x \in \gamma_{\beta}$. By Proposition 4.2, $\gamma_{\beta} \subset \operatorname{Sing} \alpha$. Remark 2.2 gives a contradiction.

In particular, the supports of collinear Morse forms (closed one-forms with nondegenerated singularities) coincide [10]. The condition for the forms to be closed is important; see Figure 3.

Recall that by Proposition 3.6, $\mathcal{F}_{\alpha}=\mathcal{F}_{\beta}$ implies $\alpha \| \beta$.

Proposition 6.4. Let $\alpha$ be a closed one-form. Then $\overline{\mathcal{F}}_{\beta}=\overline{\mathcal{F}}_{\alpha}$ implies $\beta \| \alpha$.

Proof. Let $x \in S=S(\alpha, \beta)$. Consider a curve $s(t) \subseteq \gamma_{\beta} \in \mathcal{F}_{\beta}, x \in s(t)$. Then $s(t) \subseteq$ $\tilde{\gamma}_{\beta}=\tilde{\gamma}_{\alpha} \in \overline{\mathcal{F}}_{\alpha}$ and, by Lemma 5.3, we have $\alpha(\dot{s}(t))=0$. Thus $\left\{\beta_{x}=0\right\} \subseteq\left\{\alpha_{x}=0\right\}$, then $\left\{\beta_{x}=0\right\}=\left\{\alpha_{x}=0\right\}$ and $\beta_{x} \| \alpha_{x}$. 

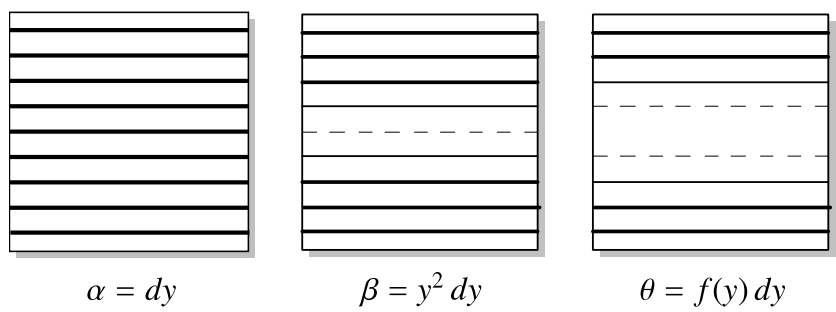

$\theta=f(y) d y$

FIGURE 8. Closed collinear one-forms on a 2-torus: $\operatorname{Sing} \alpha=\emptyset$, $\operatorname{Sing} \beta=S^{1}$, and $\operatorname{Sing} \theta=I \times S^{1}$.

Now we are ready to formulate our main result.

THEOREM 6.5. Let $\alpha$ and $\beta$ be closed forms with nowhere dense singular sets (see Lemma 2.3). Then $\overline{\mathcal{F}}_{\alpha}=\overline{\mathcal{F}}_{\beta}$ if and only if $\alpha \| \beta$.

If, moreover, $\operatorname{dim} \operatorname{Sing} \alpha \leq n-2$ and $\operatorname{dim} \operatorname{Sing} \beta \leq n-2$, then $\mathcal{F}_{\alpha}=\mathcal{F}_{\beta}$ if and only if $\alpha \| \beta$ (in particular, $\alpha \| \beta$ implies $\operatorname{Sing} \alpha=\operatorname{Sing} \beta$ ).

Proof. Let $\alpha \| \beta$. Consider a leaf $\tilde{\gamma}_{\alpha} \in \overline{\mathcal{F}}_{\alpha}$. Let $s:[0,1] \rightarrow \tilde{\gamma}_{\alpha}$ be a map, from Definition 5.2, so that $\alpha(\dot{s}(t))=0$ for all $t \in[0,1]$. Then also $\beta(\dot{s}(t))=0$.

Indeed, suppose at $s(t) \in \operatorname{Supp} \beta$, and thus in a small neighbourhood $U=$ $U(s(t)) \subset \operatorname{Supp} \beta$, the curve $s$ is transversal to the leaves of $\mathcal{F}_{\beta}$. By Lemma 3.3, on Supp $\beta$ it holds that $\alpha=f(x) \beta$, so we have $f(x)=0$ for $s \cap U$. Then Lemma 4.1 gives $f(x) \equiv 0$ in $U$, so $U \subseteq \operatorname{Sing} \alpha$; this is a contradiction. Thus $\beta(\dot{s}(t))=0$.

We obtain $\tilde{\gamma}_{\alpha} \subseteq \tilde{\gamma}_{\beta} \in \overline{\mathcal{F}}_{\beta}$ and vice versa, that is, $\overline{\mathcal{F}}_{\alpha}=\overline{\mathcal{F}}_{\beta}$. The converse follows from Proposition 6.4. Finally, $\mathcal{F}_{\alpha}=\mathcal{F}_{\beta}$ follows from Lemma 6.3 and Proposition 3.6.

Note that $\overline{\mathcal{F}}_{\alpha}=\overline{\mathcal{F}}_{\beta}$ here is not trivially true, as in Figure 7, since, by Corollary 5.4, under the conditions of the theorem it holds that $\operatorname{dim} \tilde{\gamma} \leq n-1$.

The condition for the forms to be closed is important: in Figure $4, \alpha \| \beta$ but $\overline{\mathcal{F}}_{\alpha} \neq \overline{\mathcal{F}}_{\beta}$; in Figure $7, \overline{\mathcal{F}}_{\beta}=\overline{\mathcal{F}}_{\theta}$ but $\beta \not \mid \theta$. This condition is also important for the second part of the theorem: in Figure $3, \alpha \| \beta$ and $\overline{\mathcal{F}}_{\alpha}=\overline{\mathcal{F}}_{\beta}$, but $\mathcal{F}_{\alpha} \neq \mathcal{F}_{\beta}$ because $\operatorname{Sing} \alpha \neq \operatorname{Sing} \beta$.

The condition $\operatorname{dim} \operatorname{Sing} \beta \leq n-2$ in the second part of Theorem 6.5 is important: in Figure $8, \alpha \| \beta$ are closed forms and therefore $\overline{\mathcal{F}}_{\alpha}=\overline{\mathcal{F}}_{\beta}$, but $\mathcal{F}_{\alpha} \neq \mathcal{F}_{\beta}$ because Sing $\alpha \neq \operatorname{Sing} \beta$.

Finally, the condition for the singular sets to be nowhere dense is important: in Figure 8, where Sing $\theta$ is a band, $\overline{\mathcal{F}}_{\alpha} \neq \overline{\mathcal{F}}_{\theta}$. Another example is Figure 2, right.

Theorem 6.5 describes the set of closed one-forms with small enough singular sets that define the same foliation $\mathcal{F}$, eliminating the requirement of coincidence of singular sets in Theorem 3.7.

Corollary 6.6. Let $A$ be the class of closed one-forms with $\operatorname{dim} \operatorname{Sing} \alpha \leq n-2$. Given a foliation $\mathcal{F}=\mathcal{F}_{\alpha}$ of form $\alpha \in A$, the forms from $A$ defining $\mathcal{F}$ are those collinear with $\alpha$. 
Similarly, for the class $A$ of closed one-forms with nowhere dense singular set, the forms from $A$ defining $\overline{\mathcal{F}}=\overline{\mathcal{F}}_{\alpha}$ are those collinear with $\alpha$.

Note that the only property of covering dimension used in our results regarding $\operatorname{dim} \operatorname{Sing} \alpha \leq n-2$ is Remark 2.2: if $\operatorname{dim} X \leq k$, then $X$ does not contain $\mathbb{R}^{k}$. This weaker condition can be used in Lemma 6.3, Theorem 6.5, and Corollary 6.6: instead of $\operatorname{dim} \operatorname{Sing} \alpha \leq n-2$, it is enough to require $\operatorname{Sing} \alpha$ not to contain a smooth inclusion of $\mathbb{R}^{n-1}$. This generalises these results to forms with $\operatorname{dim} \operatorname{Sing} \alpha=n-1$ whose singular set is just not smooth enough to contain $\mathbb{R}^{n-1}$, such as $\theta$ in Figure 4.

\section{Applications of collinearity: foliations of closed one-forms}

The notion of collinearity is a useful tool for studying the topology of foliations of closed one-forms.

Since a foliation defined by a closed one-form has no holonomy, by the Reeb local stability theorem, each of its compact leaves has a neighbourhood consisting of compact leaves. It can be explicitly constructed as shown in the following lemma.

LEMMA 7.1. Let $\alpha$ be a closed one-form. Then any compact leaf $\gamma \in \mathcal{F}_{\alpha}$ has a cylindrical neighbourhood consisting of compact leaves.

PROOF. Let $U$ be a neighbourhood of $\gamma$ where the form is exact: $\alpha=d f$; assume $\left.f\right|_{\gamma}=0$. For some $\varepsilon>0$, consider a neighbourhood $U_{\varepsilon}=\{x \in U|| f(x) \mid<\varepsilon\}$. Since $\operatorname{Supp} \alpha$ is open, we can choose $\varepsilon$ such that $U_{\varepsilon} \cap \operatorname{Sing} \alpha=\emptyset$. Obviously, $\gamma \subset U_{\varepsilon}$. By construction,

$$
U_{\varepsilon}=\bigcup_{|y|<\varepsilon} \gamma_{y} \simeq \gamma \times(-\varepsilon, \varepsilon),
$$

where $\gamma_{y}=f^{-1}(y)$ are compact leaves of $\mathcal{F}_{\alpha}$.

By choosing a suitable form $\beta \| \alpha$, one can characterise the topology of the foliation $\mathcal{F}_{\alpha}$. For example, if there exists an exact form $d f \| \alpha$ with $\operatorname{Supp} d f=\operatorname{Supp} \alpha$, then the foliation $\mathcal{F}_{\alpha}$ is compactifiable, that is, for any leaf $\gamma \in \mathcal{F}_{\alpha}$, the set $\gamma \cup \operatorname{Sing} \alpha$ is compact. Indeed, the foliation $\mathcal{F}_{d f}=\mathcal{F}_{\alpha}$ is defined by levels of the function $f(x)$, which are compact.

Farber et al. [4, 5] gave a necessary condition for the existence of a compact leaf in the foliation defined by a so-called transitive Morse form in terms of the cup-product: if $\mathcal{F}_{\alpha}$ has a compact leaf $\gamma,[\gamma] \neq 0$, then there exists a closed one-form $\beta, 0 \neq[\beta] \in$ $H^{1}(M, \mathbb{Z})$ such that $[\alpha] \smile[\beta]=0$. This is not a criterion; moreover, no sufficient condition for the existence of a compact leaf can be given in purely cohomologous terms since in any cohomology class $[\alpha]$, rk $\alpha>1$, there exists a form with minimal foliation [1]; rk $\alpha$ is the rank of its group of periods (integrals over one-cycles).

We generalise this condition in terms of collinearity to an arbitrary closed one-form and improve it to a criterion.

THEOREM 7.2. Let $\alpha$ be a closed one-form. The following conditions are equivalent.

$\mathcal{F}_{\alpha}$ has a compact leaf $\gamma$. 
(ii) There exists a smooth function $f(x)$ such that:

(a) $d f \| \alpha$;

(b) $\operatorname{Supp} \alpha \cap \operatorname{Supp} d f \neq \varnothing$.

(iii) There exists a smooth closed one-form $\beta$ such that:

(a) $\beta \| \alpha$;

(b) $\operatorname{Supp} \alpha \cap \operatorname{Supp} \beta \neq \emptyset$;

(c) $[\beta] \in H^{1}(M, \mathbb{Z})$.

Given a specific $\gamma, f$, or $\beta$, the other two can be chosen such that $\left.f\right|_{\gamma}=$ const and $[\beta]=D[\gamma]$, where $D: H_{n-1}(M) \rightarrow H^{1}(M, \mathbb{Z})$ is the Poincaré duality map.

PROOF. (i) $\Rightarrow$ (ii). Consider the function from Lemma 7.1, defined on a cylindrical neighbourhood $\gamma \times(-\varepsilon / 2, \varepsilon / 2)$ consisting of compact diffeomorphic leaves, and extend it to a smooth function $f$, constant on leaves, such that $f \equiv 0$ outside $\gamma \times(-\varepsilon, \varepsilon)$. Then $\operatorname{Supp} \alpha \cap \operatorname{Supp} d f \neq \emptyset$. By Proposition 3.6, $d f \| \alpha$.

(ii) $\Rightarrow$ (iii). Consider $\beta=d f ;[\beta]=0$.

(iii) $\Rightarrow$ (i). Since $[\beta] \in H^{1}(M, \mathbb{Z})$, the form $\beta$ defines a map $F: M \rightarrow S^{1}$,

$$
F(x)=e^{2 \pi i \int_{x_{0}}^{x} \beta} .
$$

Obviously, $F$ is constant on leaves of $\mathcal{F}_{\beta}$ and the critical set of $F$ coincides with $\operatorname{Sing} \beta$. Consider $F_{S}=\left.F\right|_{S}: S \rightarrow S^{1}$, where $S=\operatorname{Supp} \alpha \cap \operatorname{Supp} \beta$.

By Sard's theorem, the map $F_{S}$ has a regular value $y \in S^{1}$, and by the implicit function theorem, $F_{S}^{-1}(y)$ is a closed codimension-one submanifold. By Corollary 4.3, the set $S$ consists of entire leaves of both $\mathcal{F}_{\alpha}$ and $\mathcal{F}_{\beta}$, so a connected component of $F_{S}^{-1}(y)$ is a compact leaf $\gamma \in \mathcal{F}_{\beta} \cap \mathcal{F}_{\alpha}$. Thus (iii) $\Rightarrow$ (i).

Now, given a specific $\gamma$, the function $f$ constructed above satisfies $\left.f\right|_{\gamma}=$ const. Let us construct $\beta$ such that $[\beta]=D[\gamma]$.

Construct the function $f$ as above; denote $U=\gamma \times(-\varepsilon, \varepsilon)$. Consider a curve $s(t) \subset U, t \in(-\varepsilon, \varepsilon)$, transversal to leaves. Obviously, the form $f(x) \alpha$ is closed; denote $A=\int_{-\varepsilon}^{\varepsilon} f(s(t)) \alpha(\dot{s}(t)) d t$, which is finite. Let $\beta=(1 / A) f(x) \alpha$. This form is closed and collinear with $\alpha$; by construction, $\operatorname{Supp} \alpha \cap \operatorname{Supp} \beta \neq \emptyset$.

Consider an arbitrary $(n-1)$-form $\theta$. Since Supp $\beta \subseteq U$ and $\int_{-\varepsilon}^{\varepsilon} \beta(\dot{s}(t)) d t=1$,

$$
\int_{M} \theta \wedge \beta=\int_{U} \theta \wedge \beta=\int_{\gamma} i^{*} \theta \int_{-\varepsilon}^{\varepsilon} \beta(\dot{s}(t)) d t=\int_{\gamma} i^{*} \theta,
$$

where $i: \gamma \hookrightarrow M$ is the inclusion map. This means that $[\beta]=D[\gamma][3,(5.13)]$.

Given $f$, the leaf $\gamma$ constructed in (ii) $\Rightarrow$ (iii) and (iii) $\Rightarrow$ (i) satisfies $\left.f\right|_{\gamma}=$ const; then a new $\beta$ is constructed as above. Given $\beta$, the leaf $\gamma$ constructed as in (iii) $\Rightarrow$ (i) and $f$ constructed in (i) $\Rightarrow$ (ii) satisfy the conditions.

Finally, let us show that homological properties of compact leaves are connected with the structure of the cup-product $\smile: H^{1}(M, \mathbb{Z}) \times H^{1}(M, \mathbb{Z}) \rightarrow H^{2}(M, \mathbb{Z})$. 
Denote by $c(\alpha)$ the maximum number of homologically independent compact leaves of $\mathcal{F}_{\alpha}$. This value plays an important role in the theory of Morse form foliations [14-16]; in particular, in this case

$$
c(\alpha)+m(\alpha) \leq \begin{cases}b_{1}^{\prime}(M) & {[8],} \\ b_{1}(M) / 2 & {[9],} \\ h(M) & {[9],}\end{cases}
$$

where $m(\alpha)$ is the number of minimal components of $\mathcal{F}_{\alpha}, b_{1}^{\prime}(M)$ is the first noncommutative Betti number [1], and $h(M)$ is the maximum rank of a subgroup in $H^{1}(M, \mathbb{Z})$ with trivial cup-product [17], an important characteristic of the manifold [7].

For a closed one-form $\alpha$, its rank rk $\alpha$ is the rank of its group of periods:

$$
\operatorname{rk} \alpha=\operatorname{rk}_{\mathbb{Q}}\left\{\int_{z} \alpha \mid z \in H_{1}(M)\right\} .
$$

Obviously, $0 \leq \mathrm{rk} \alpha \leq b_{1}(M)$, the first Betti number.

For an arbitrary closed one-form, obviously, $c(\alpha) \leq h(M)$. For forms of the maximum possible rank for the given $M$, a stronger fact holds (shown in [10] for Morse forms).

TheOREM 7.3. Assume rk $\alpha=b_{1}(M)$. Then $c(\alpha) \leq$ rk ker $\smile$.

PROOF. For $c(\alpha)=0$ the statement is trivial, so assume there exists a homologically nontrivial compact leaf $\gamma \in \mathcal{F}_{\alpha},[\gamma] \neq 0$. By Theorem 7.2, there exists a smooth closed one-form $\beta \| \alpha$ such that $[\beta]=D[\gamma] \in H^{1}(M, \mathbb{Z})$. Since $\beta \wedge \alpha=0$, we have $[\beta] \smile_{\mathbb{R}}[\alpha]=\sum \alpha_{i}\left([\beta] \smile \xi_{i}\right)=0$, where $\smile_{\mathbb{R}}$ is the cup-product on $H^{1}(M, \mathbb{R}),\left\{\xi_{i}\right\}$ is a basis in $H^{1}(M, \mathbb{Z})$, and $[\alpha]=\sum \alpha_{i} \xi_{i}$.

Denote $u_{i}=[\beta] \smile \xi_{i} ; \sum \alpha_{i} u_{i}=0$. Since rk $\alpha=b_{1}(M)$, all $\alpha_{i}$ are independent over $\mathbb{Q}$. Thus all $u_{i}$ belong to the torsion of $H^{2}(M, \mathbb{Z})$, that is, for some $0 \neq k \in \mathbb{Z}$, we have $k[\beta] \smile \xi_{i}=0$ for all $i$, so $k[\beta] \in \operatorname{ker} \smile$. Since $H^{1}(M, \mathbb{Z})$ has no torsion, $k[\beta] \neq 0$.

Now consider $c(\alpha)$ homologically independent compact leaves $\gamma_{1}, \ldots, \gamma_{c(\alpha)}$ and the corresponding $\beta_{i}$ as above such that $k_{i}\left[\beta_{i}\right] \in \operatorname{ker} \smile$ for some $0 \neq K_{i} \in \mathbb{Z}$. Since the $\left[\gamma_{1}\right], \ldots,\left[\gamma_{c(\alpha)}\right]$ are independent, so are $\left[\beta_{i}\right]=D\left[\gamma_{i}\right]$, and therefore $k_{i}\left[\beta_{i}\right]$; thus rk ker $\smile \geq c(\alpha)$.

\section{References}

[1] P. Arnoux and G. Levitt, 'Sur l'unique ergodicité des 1-formes fermées singulières', Invent. Math. 84 (1986), 141-156.

[2] E. Ayón-Beato, A. García, A. Macías and H. Quevedo, 'Static black holes of metric-affine gravity in the presence of matter', Phys. Rev. D 64 (2001), 024026.

[3] R. Bott and L. W. Tu, Differential Forms in Algebraic Topology, Graduate Texts in Mathematics, 82 (Springer, New York, 1982). 
[4] M. Farber, Topology of Closed One-forms, Mathematical Surveys, 108 (American Mathematical Society, Providence, RI, 2004).

[5] M. Farber, G. Katz and J. Levine, 'Morse theory of harmonic forms', Topology 37(3) (1998), 469-483.

[6] J. J. Ferrando and J. A. Sáez, 'Type I vacuum solutions with aligned Papapetrou fields: an intrinsic charactrization', J. Math. Phys. 47 (2006), 112501.

[7] I. Gelbukh, 'Presence of minimal components in a Morse form foliation', Differential Geom. Appl. 22 (2005), 189-198.

[8] I. Gelbukh, 'Number of minimal components and homologically independent compact leaves for a Morse form foliation', Studia Sci. Math. Hungar. 46(4) (2009), 547-557.

[9] I. Gelbukh, 'On the structure of a Morse form foliation', Czechoslovak Math. J. 59(1) (2009), 207-220.

[10] I. Gelbukh, 'Ranks of collinear Morse forms', J. Geom. Phys. 61(2) (2011), 425-435.

[11] F. W. Hehl and J. Socorro, 'Gauge theory of gravity: electrically charged solutions within the metric-affine framework', Acta Phys. Polon. B 29 (1998), 1113-1120.

[12] W. Hurewicz and H. Wallman, Dimension Theory (Princeton University Press, Princeton, NJ, 1996).

[13] D. W. Kahn, Introduction to Global Analysis, Pure and Applied Mathematics, 91 (Academic Press, New York, 1980).

[14] I. Mel'nikova, 'A test for compactness of a foliation', Math. Notes 58(6) (1995), 1302-1305.

[15] I. Mel'nikova, 'Singular points of a Morsian form and foliations', Moscow Univ. Math. Bull. 51(4) (1996), 33-36.

[16] I. Mel'nikova, 'Noncompact leaves of foliations of Morse forms', Math. Notes 63(6) (1998), $760-763$.

[17] I. Mel'nikova, 'Maximal isotropic subspaces of skew-symmetric bilinear mapping', Moscow Univ. Math. Bull. 54(4) (1999), 1-3.

[18] S. B. Mulay, 'Cycles and symmetries of zero-divisors', Comm. Algebra 30 (2002), 3533-3558.

[19] R. Sacksteder, 'Foliations and pseudo-groups', Amer. J. Math. 87 (1965), 79-102.

IRINA GELBUKH, Waseda Step 21, Room 403, Totsuka-Machi 1-103, Shinjuku-ku, Tokyo 169-0071, Japan e-mail: gelbukh@member.ams.org 